\title{
TOLERÂNCIA DE PLANTAS DE Khaya ivorensis E Toona ciliata A HERBICIDAS
}

\author{
Alexandre Magno Brighenti ${ }^{1}$, Marcelo Dias Muller ${ }^{2}$ \\ ${ }^{1}$ Eng. Agrônomo, Dr., EMBRAPA Gado de Leite, Juiz de Fora, MG, Brasil - alexandre.brighenti@embrapa.br \\ ${ }^{2}$ Eng. Florestal, Dr., EMBRAPA Gado de Leite, Juiz de Fora, MG, Brasil - marcelo.muller@ embrapa.br
}

Recebido para publicação: 29/05/2013 - Aceito para publicação: 28/04/2014

\begin{abstract}
Resumo
Dois experimentos foram conduzidos com o objetivo de avaliar a tolerância de plantas mognoafricano (Khaya ivorensis) e cedro-australiano (Toona ciliata var. australis) a herbicidas. O delineamento experimental foi inteiramente casualizado, com quatro repetições. Tratamentos: i) testemunha sem aplicação, ii) imazapyr (25 g i.a. ha $\left.{ }^{-1}\right)$, iii) oxyfluorfen (360 g i.a. ha $\left.{ }^{-1}\right)$, iv) oxyfluorfen $\left(720 \mathrm{~g}\right.$ i.a. ha $\left.{ }^{-1}\right)$, v) isoxaflutole $\left(75 \mathrm{~g}\right.$ i.a. ha $\left.{ }^{-1}\right)$, vi) isoxaflutole $\left(112,5 \mathrm{~g} \mathrm{i.a.} \mathrm{ha}{ }^{-1}\right)$, vii) chlorimuron-ethyl $\left(7,5 \mathrm{~g}\right.$ i.a. ha $\left.{ }^{-1}\right)+0,05 \% \mathrm{v} / \mathrm{v}$ de óleo mineral, viii) chlorimuron-ethyl (12,5 g i.a. $\left.\mathrm{ha}^{-1}\right)+0,05 \%$ v/v de óleo mineral, ix $)$ imazethapyr $\left(100 \mathrm{~g}\right.$ i.a. ha $\left.{ }^{-1}\right)$ e x $)$ nicosulfuron $\left(40 \mathrm{~g} \mathrm{i} . \mathrm{a} . \mathrm{ha}^{-1}\right)$. Para o mogno africano, os herbicidas imazapyr $\left(25 \mathrm{~g}\right.$ i.a. ha $\left.{ }^{-1}\right)$, oxyfluorfen $\left(360 \mathrm{~g}\right.$ i.a. ha $\left.{ }^{-1}\right)$ e chlorimuron-ethyl $\left(7,5 \mathrm{e} 12,5 \mathrm{~g}\right.$ i.a. ha $\left.^{-1}\right)$ proporcionaram sintomas leves de fitotoxicidade. O oxyfluorfen (720 g i.a. ha ${ }^{-1}$ ), o imazethapyr e o nicosulfuron foram moderadamente fitotóxicos e o isoxaflutole (75 e 112,5 g i.a. ha ${ }^{-1}$ ) foi o herbicida mais fitotóxico. Para o cedro australiano, o herbicida imazapyr (25 g i.a. ha $^{-1}$ ) proporcionou os menores níveis de injúria. O oxyfluorfen, o isoxaflutole, o chlorimuron-ethyl, o imazethapyr e o nicosulfuron proporcionaram altos níveis de fitotoxicidade.

Palavras-chave: Mogno africano; cedro australiano; plantas daninhas; seletividade.
\end{abstract}

\begin{abstract}
Tolerance of Khaya ivorensis and Toona ciliata to herbicides. We conducted two experiments to evaluate the plant tolerance of African mahogany (Khaya ivorensis) and Australian cedar (Toona ciliata var. australis) to herbicides. The experimental arrangement was a completely randomized design, with four replications. The treatments were as follows: i) untreated control, ii) imazapyr ( $25 \mathrm{~g}$ ai ha $\left.{ }^{-1}\right)$, iii) oxyfluorfen $\left(360 \mathrm{~g}\right.$ ai ha $\left.^{-1}\right)$, iv) oxyfluorfen $\left(720 \mathrm{~g}\right.$ ai ha $\left.\left.{ }^{-1}\right), \mathrm{v}\right)$ isoxaflutole $\left(75 \mathrm{~g}\right.$ ai ha $\left.{ }^{-1}\right)$, vi) isoxaflutole $\left(112.5 \mathrm{~g}\right.$ ai ha $\left.{ }^{-1}\right)$, vii) chlorimuron-ethyl $\left(7.5 \mathrm{~g} \mathrm{ai} \mathrm{ha}^{-1}\right)+0.05 \% \mathrm{v} / \mathrm{v}$ mineral oil, viii) chlorimuron-ethyl $\left(12.5 \mathrm{~g}_{\text {ai ha }}{ }^{-1}\right)+0.05 \% \mathrm{v} / \mathrm{v}$ mineral oil, ix $)$ imazethapyr (100 g ai ha $\left.{ }^{-1}\right)$, and $\left.\mathrm{x}\right)$ nicosulfuron (40 $\left.\mathrm{g}_{\text {ai }} \mathrm{ha}^{-1}\right)$. For the African mahogany, imazapyr (25 $\mathrm{g}$ ai ha $\left.{ }^{-1}\right)$, oxyfluorfen (360 $\mathrm{g}$ ai $\mathrm{ha}^{-1}$ ) and chlorimuron-ethyl (7.5 and $12.5 \mathrm{~g}$ ai ha $\left.{ }^{-1}\right)$, showed mild symptoms of phytotoxicity. The oxyfluorfen (720 g ai ha $\left.{ }^{-1}\right)$, imazethapyr and nicosulfuron were moderately phytotoxics, and

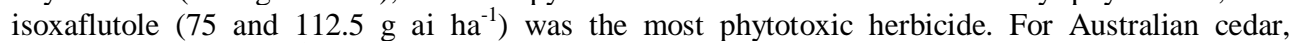
imazapyr (25 $\mathrm{g}$ ai ha ${ }^{-1}$ ) caused low levels of injury. Oxyfluorfen, isoxaflutole, chlorimuron-ethyl, imazethapyr and nicosulfuron were the most phytotoxic herbicides.

Keywords: African mahogany; Australian cedar; weeds; selectivity.
\end{abstract}

\section{INTRODUÇÃO}

A incorporação do conceito de sustentabilidade tem levado a pesquisa agropecuária a buscar modelos alternativos e sustentáveis de produção agrícola e ainda à necessidade de desenvolvimento de técnicas capazes de reduzir as quantidades de produtos fitossanitários utilizados no campo, exigindo padrões de qualidade dos alimentos consumidos e a preservação do ambiente como um todo.

Nesse âmbito, o sistema de Integração Lavoura-Pecuária-Floresta (iLPF) vem contribuir para minimizar os efeitos provocados pela agricultura tradicional. Essa tecnologia é definida como diferentes sistemas produtivos de grãos, fibras, madeira, carne, leite e agroenergia, implantados na mesma área em consórcio, em rotação ou em sucessão, envolvendo a semeadura de grãos, de pastagens e de cultivos arbóreos associados (BALBINO et al., 2011). 
Uma característica importante desses sistemas são os espaçamentos mais amplos adotados entre as árvores, a fim de proporcionar maior incidência de luz no sub-bosque e consequentemente favorecer o desenvolvimento do pasto. Entretanto, além das espécies forrageiras, tais condições favorecem o desenvolvimento de plantas infestantes (FERREIRA et al., 2012). Dessa forma, um dos fatores de sucesso na implantação de sistemas de iLPF está relacionado ao manejo de plantas daninhas (FERREIRA et al., 2010). Contudo, com o surgimento de alternativas de espécies arbóreas com potencial de inserção em sistemas integrados, há carência de informações relacionadas à tolerância dessas espécies a herbicidas.

Quando se observam os trabalhos relacionados ao componente florestal em iLPF, a maioria deles vem sendo conduzida com eucalipto e pouca informação existe sobre outras espécies potencialmente capazes de fazer parte do sistema (BRIGHENTI et al., 2007).

Com o aumento crescente da utilização de madeira de reflorestamentos para serraria, cresce também o interesse por espécies florestais alternativas, que detêm características tecnológicas favoráveis para esse uso. Como exemplo, há o mogno-africano (Khaya ivorensi A. Chev.) e o cedro-australiano (Toona ciliata M. Roem. var. australis). O mogno-africano possui madeira de qualidade, com elevado valor econômico internacional, dada a sua beleza e durabilidade, além da excelente usinagem da madeira (CARVALHO et al., 2010; PINHEIRO et al., 2011). No caso do cedro-australiano, a madeira também é considerada de alta qualidade e própria para fabricação de produtos de alto valor agregado, como móveis finos, laminados, forros, molduras, uso naval e instrumentos musicais (PINHEIRO et al., 2003; MULLER, 2004; ALBINO et al., 2010; SÁ et al., 2010).

Com a necessidade crescente de introdução de espécies florestais alternativas nos sistemas de iLPF, alguns entraves, como, por exemplo, o manejo de plantas daninhas, necessita ser minuciosamente estudado. Além disso, o setor florestal tem se expandido, principalmente, para áreas do cerrado, as quais são ocupadas, na sua grande maioria, por pastagens, principalmente de braquiárias. Essas espécies, devido a sua agressividade, estão entre as plantas mais competitivas nos plantios florestais (TOLEDO et al., 1996; TOLEDO et al., 2000a), sendo os dois primeiros anos após a implantação aqueles de maior atenção no que se refere ao controle de espécies daninhas (TOLEDO et al., 2000b).

Nesse contexto, os estudos de seletividade de herbicidas são importantes para o sucesso do controle químico de plantas infestantes. Quanto maior a diferença de tolerância entre a cultura e as plantas daninhas, maior a segurança de aplicação dos herbicidas (OLIVEIRA JR.; INOUE, 2011). Esses estudos são também importantes no sentido de contribuir nas tomadas de decisão relacionadas ao registro e recomendações dos órgãos competentes.

O objetivo deste trabalho foi avaliar a tolerância de plantas de mogno-africano e cedroaustraliano a herbicidas, a fim de dar subsídios ao manejo de espécies daninhas nesses cultivos.

\section{MATERIAL E MÉTODOS}

Dois experimentos foram instalados no município de Coronel Pacheco, MG $\left(21^{\circ} 32^{\prime} 17^{\prime \prime} \mathrm{S}\right.$ e $43^{\circ} 14^{\prime} 30^{\prime \prime}$ W). O clima da região é do tipo Cwa (mesotérmico), definido como subtropical, chuvoso no verão e seco no inverno. A pluviosidade média anual é de aproximadamente $1.500 \mathrm{~mm}$.

$\mathrm{O}$ delineamento experimental foi o inteiramente casualizado, com quatro repetições. Cada unidade experimental foi constituída por uma planta por vaso. Os tratamentos foram: i) testemunha sem aplicação, ii) imazapyr $\left(25 \mathrm{~g}\right.$ i.a. ha $\left.{ }^{-1}\right)$, iii) oxyfluorfen $\left(360 \mathrm{~g}^{-1}\right.$ i.a. ha $\left.\mathrm{g}^{-1}\right)$, iv) oxyfluorfen $\left(720 \mathrm{~g} \mathrm{i}^{\mathrm{a} . \mathrm{ha}^{-1}}\right)$, v) isoxaflutole (75 g i.a. ha $\left.{ }^{-1}\right)$, vi) isoxaflutole $\left(112,5 \mathrm{~g}\right.$ i.a. ha $\left.\mathrm{h}^{-1}\right)$, vii) chlorimuron-ethyl $\left(7,5 \mathrm{~g}\right.$ i.a. $\left.\mathrm{ha}^{-1}\right)+$ $0,05 \% \mathrm{v} / \mathrm{v}$ de óleo mineral, viii) chlorimuron-ethyl $\left(12,5 \mathrm{~g}\right.$ i.a. $\left.\mathrm{ha}^{-1}\right)+0,05 \% \mathrm{v} / \mathrm{v}$ de óleo mineral, ix) imazethapyr (100 g i.a. ha $\left.{ }^{-1}\right)$ e $\left.\mathrm{x}\right)$ nicosulfuron $\left(40 \mathrm{~g}^{\mathrm{i}}\right.$.a. $\left.\mathrm{ha}^{-1}\right)$.

Os experimentos foram implantados em vasos de $10 \mathrm{~kg}$ de capacidade e dispostos em bancadas em ambiente natural. O material para enchimento dos vasos foi composto de três partes de solo (Latossolo Vermelho-Amarelo), duas partes de esterco e uma de areia. Sementes de mogno-africano (Khaya ivorensis) (experimento 1) e cedro-australiano (Toona ciliata) (experimento 2) foram plantadas previamente em tubetes e transplantadas para os vasos em 14/02/2011, com altura aproximada de $20 \mathrm{~cm}$ e 4 pares de folhas. Na época do plantio, foi colocada, em cada vaso, uma quantidade de $80 \mathrm{~g}$ da formulação NPK (4-30-10) e $10 \mathrm{~g}$ de bórax.

A aplicação dos herbicidas foi realizada em 18/04/2011, utilizando-se um pulverizador de pesquisa (Herbicat, Catanduva, SP), mantido a pressão constante de $\mathrm{CO}_{2}$ comprimido, equivalente a $2 \mathrm{kgf.cm}{ }^{-2}$. A barra de pulverização era composta de dois bicos de jato plano AVI 110 015, distanciados 


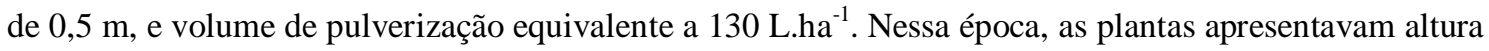
média de $40 \mathrm{~cm}$ e 6 pares de folhas.

Foram avaliados o percentual de fitotoxicidade das plantas aos 7, 14 e 21 DAA (dias após a aplicação dos tratamentos), em que zero correspondeu a nenhum sintoma visual de injúria e $100 \%$ a morte total das plantas (SOCIEDADE BRASILEIRA DA CIÊNCIA DAS PLANTAS DANINHAS, 2005). Foi adotado como critério de seleção um limite máximo de fitotoxicidade de 5\%, aos 21 DAA, ou seja, o dano máximo para considerar o produto e a dose seletivos.

Foram contados o número das folhas das plantas aos 21 e 28 DAA e ainda obtidos os teores de clorofila (índice SPAD) das folhas aos 21 dias após a aplicação dos tratamentos, por meio do medidor de clorofila SPAD 502, Konica Minolta (Konica Minolta Sensing Inc., Japão). Em cada planta, foram obtidas cinco medições de cinco folhas a partir do terço médio em direção ao ápice.

Para o cedro-australiano, foram obtidos os valores de área foliar utilizando-se o medidor de área foliar marca LICOR 3100. Além disso, as plantas coletadas foram dispostas em estufa de ventilação forçada de ar a $60{ }^{\circ} \mathrm{C}$, até atingirem massa constante. Em seguida, foram obtidos os pesos da matéria seca de raízes e de caules e a matéria seca total.

Os dados foram submetidos à análise de variância e as médias comparadas pelo teste Scott-Knott $(\mathrm{p} \leq 0,05)$.

\section{RESULTADOS E DISCUSSÃO}

Os dados para o experimento 1 relativos à percentagem de fitotoxicidade aos 7, 14 e 21 DAA, do número de folhas aos 21 e aos 28 DAA e do teor de clorofila (índice SPAD) aos 21 DAA, em função da aplicação dos herbicidas, encontram-se na tabela 1.

Tabela 1. Percentagem de fitotoxicidade aos 7, 14 e 21 DAA (dias após a aplicação dos herbicidas), número de folhas aos $21 \mathrm{DAA}\left(\mathrm{NF}_{1}\right)$ e aos $28 \mathrm{DAA}\left(\mathrm{NF}_{2}\right)$ e teor de clorofila (índice $\left.\mathrm{SPAD}\right)$ aos 21 DAA, em função da aplicação das doses dos herbicidas sobre mogno-africano.

Table 1. Percentage of phytotoxicity at 7,14 and 21 DAA (days after herbicide application), number of leaves at 21 DAA (NF1) and at 28 DAA (NF2), and chlorophyll content (SPAD index) at 21 DAA, in function of the application of herbicides on African mahogany.

\begin{tabular}{|c|c|c|c|c|c|c|c|}
\hline & 7DAA & 14DAA & 21DAA & $\mathrm{NF}_{1}$ & $\mathrm{NF}_{2}$ & SPAD \\
\hline \multicolumn{2}{|l|}{ Testemunha } & $0,0 \mathrm{~d}$ & $0,0 \mathrm{e}$ & $0,0 \mathrm{e}$ & $30,3 \mathrm{~b}$ & $34,0 \mathrm{~b}$ & $55,2 \mathrm{a}$ \\
\hline Imazapyr & 25 g i.a. ha ${ }^{-1}$ & $0,0 \mathrm{~d}$ & $0,0 \mathrm{e}$ & $0,0 \mathrm{e}$ & $25,6 \mathrm{~b}$ & $25,3 \mathrm{~b}$ & $40,8 \mathrm{~b}$ \\
\hline Oxyfluorfen & 360 g i.a. ha ${ }^{-1}$ & $0,0 \mathrm{~d}$ & $0,0 \mathrm{e}$ & $5,0 \mathrm{~d}$ & $17,3 \mathrm{~b}$ & $18,0 \mathrm{~b}$ & $54,3 \mathrm{a}$ \\
\hline Oxyfluorfen & 720 g i.a. ha ${ }^{-1}$ & $10,0 \mathrm{c}$ & $10,0 \mathrm{c}$ & $6,3 \mathrm{c}$ & $41,0 \mathrm{a}$ & $52,3 \mathrm{a}$ & $56,7 \mathrm{a}$ \\
\hline Isoxaflutole & 75 g i.a. ha ${ }^{-1}$ & $15,0 \mathrm{~b}$ & $20,0 \mathrm{~b}$ & 15,3 a & $28,0 \mathrm{~b}$ & $45,3 \mathrm{a}$ & $55,1 \mathrm{a}$ \\
\hline Isoxaflutole & 112,5 g i.a. $\mathrm{ha}^{-1}$ & $21,6 \mathrm{a}$ & $28,6 \mathrm{a}$ & $15,3 \mathrm{a}$ & $52,0 \mathrm{a}$ & $51,0 \mathrm{a}$ & $54,3 \mathrm{a}$ \\
\hline Chlorimuron & 7,5 g i.a. ha ${ }^{-1}$ & $0,0 \mathrm{~d}$ & $0,0 \mathrm{e}$ & $0,0 \mathrm{e}$ & $33,3 \mathrm{~b}$ & $36,6 \mathrm{~b}$ & $43,9 \mathrm{~b}$ \\
\hline Chlorimuron & 12,5 g i.a. ha ${ }^{-1}$ & $0,0 \mathrm{~d}$ & $0,0 \mathrm{e}$ & $0,0 \mathrm{e}$ & $29,3 \mathrm{~b}$ & $29,0 \mathrm{~b}$ & $47,1 \mathrm{~b}$ \\
\hline Imazethapyr & 100 g i.a. ha ${ }^{-1}$ & $0,0 \mathrm{~d}$ & $5,0 \mathrm{~d}$ & $5,6 \mathrm{~d}$ & $25,0 \mathrm{~b}$ & $22,3 b$ & $47,6 \mathrm{~b}$ \\
\hline Nicosulfuron & 40 g i.a. ha ${ }^{-1}$ & $0,0 \mathrm{~d}$ & $5,0 \mathrm{~d}$ & $7,6 \mathrm{~b}$ & $19,3 \mathrm{~b}$ & $19,3 \mathrm{~b}$ & $47,0 \mathrm{~b}$ \\
\hline $\mathrm{CV}(\%)$ & & 19,5 & 5,3 & 8,1 & 25,9 & 26,3 & 7,6 \\
\hline
\end{tabular}

Médias seguidas da mesma letra em cada coluna não diferem pelo teste Scott-Knott, $\mathrm{p} \leq 0,05$.

As plantas de mogno-africano submetidas à dose reduzida de imazapyr $\left(25 \mathrm{~g}\right.$ i.a. ha $\left.{ }^{-1}\right)$ não apresentaram sintomas visuais de injúria em nenhuma das avaliações. Também não se verificaram reduções do número de folhas em relação à testemunha, em ambas as avaliações. Somente o teor de clorofila foi estatisticamente inferior à testemunha sem aplicação. As duas doses de oxyfluorfen provocaram necroses das folhas do mogno, caracterizadas por pequenas manchas amarronzadas sobre o limbo foliar. A maior dose causou sintomas mais pronunciados e verificados principalmente nas duas primeiras avaliações. Entretanto, houve recuperação das plantas, com lançamento de brotações vigorosas e novas emissões de folhas que não mais apresentavam os sintomas do herbicida. Esses dados estão de acordo com os obtidos por Silva et al. (1994) na cultura do eucalipto. Os autores observaram que o oxyflorfen causou clorose e necrose em folhas de Eucaliptus grandis Hill, E. saligna Smith e E. camaldulensis Dehnh, principalmente nas folhas mais novas e no momento da aplicação. Entretanto 
não houve comprometimento do crescimento e do desenvolvimento das plantas, não inviabilizando o uso do oxyflorfen em pré-emergência das plantas daninhas e após o transplante das mudas de eucalipto (SILVA et al., 1995).

O isoxaflutole, em ambas as doses, foi o herbicida que provocou maiores percentuais de fitotoxicidade, variando de 15 a $28 \%$. Os sintomas foram caracterizados por encarquilhamento das folhas do ápice das plantas. Entretanto, além de não ter ocorrido queda drástica de folhas, o número de folhas e os teores de clorofila foram altos em relação aos demais tratamentos. O isoxaflutole pertence ao grupo dos herbicidas inibidores da biossíntese de carotenoides, que são essenciais na proteção da clorofila contra a degradação pela luz solar (RODRIGUES; ALMEIDA, 2005). Como sintomatologia, ocorre o branqueamento dos tecidos fotossintéticos. Entretanto, esse sintoma não foi verificado nas plantas de mogno-africano. Agostinetto et al. (2010) avaliaram o efeito do isoxaflutole em plantas de eucalipto. Nesse caso, foi verificado que as espécies de Eucaliptus globulus Labill e E. saligna foram tolerantes ao herbicida. Devido a sua excelente seletividade, o isoxaflutole é recomendado para o controle de plantas daninhas em cultivos de eucalipto (RODRIGUES; ALMEIDA, 1998).

As duas doses de chlorimuron não proporcionaram sintomas visuais de fitotoxicidade em nenhuma das avaliações e também não houve redução no número de folhas, embora os teores de clorofila tenham sido estatisticamente inferiores à testemunha sem aplicação. Os tratamentos com imazethapyr e nicosulfuron foram moderadamente fitotóxicos. O limbo foliar das plantas apresentava leve clorose, mesmo na última avaliação, aos 21 DAA. Esse fato foi confirmado em função dos menores valores de índice SPAD em relação à testemunha. Tiburcio (2010) avaliou o efeito do nicosulfuron em eucalipto e verificou que esse herbicida causou valores altos de fitotoxicidade em alguns clones, limitando o incremento em área foliar e matéria seca da parte aérea das plantas.

Os dados para o experimento 2 relativos à percentagem de fitotoxicidade aos 7, 14 e 21 DAA, do número de folhas aos 21 e aos 28 DAA e do teor de clorofila aos 21 DAA, em função da aplicação dos herbicidas, encontram-se tabela 2.

Tabela 2. Percentagem de fitotoxicidade aos 7, 14 e 21 DAA (dias após a aplicação dos herbicidas), número de folhas aos $21 \mathrm{DAA}\left(\mathrm{NF}_{1}\right)$ e aos $28 \mathrm{DAA}\left(\mathrm{NF}_{2}\right)$ e teor de clorofila (índice $\mathrm{SPAD}$ ) aos 21 DAA em função da aplicação das doses dos herbicidas sobre cedro-australiano.

Table 2. Percentage of phytotoxicity at 7,14 and 21 DAA (days after herbicide application), number of leaves at 21 DAA (NF1) and at 28 DAA (NF2), and chlorophyll content (SPAD index) at 21 DAA, in function of the application of herbicides on Australian cedar.

\begin{tabular}{|c|c|c|c|c|c|c|c|}
\hline \multicolumn{2}{|l|}{ Tratamentos } & 7DAA & 14DAA & 21DAA & $\mathbf{N F}_{1}$ & $\mathbf{N F}_{2}$ & SPAD \\
\hline \multicolumn{2}{|l|}{ Testemunha } & $0,0 \mathrm{f}$ & $0,0 \mathrm{~g}$ & $0,0 \mathrm{i}$ & $169,2 \mathrm{a}$ & $153,0 \mathrm{a}$ & $37,2 \mathrm{~b}$ \\
\hline Imazapyr & 25 g i.a. ha ${ }^{-1}$ & $5,0 \mathrm{e}$ & $0,0 \mathrm{~g}$ & $0,0 \mathrm{i}$ & $138,0 \mathrm{a}$ & $129,0 \mathrm{a}$ & $42,3 \mathrm{a}$ \\
\hline Oxyfluorfen & 360 g i.a.ha $^{-1}$ & $47,5 \mathrm{~b}$ & $52,0 \mathrm{~b}$ & $35,0 \mathrm{e}$ & $76,0 \mathrm{~b}$ & $80,7 \mathrm{~b}$ & $43,6 \mathrm{a}$ \\
\hline Oxyfluorfen & 720 g i.a. ha ${ }^{-1}$ & $55,0 \mathrm{a}$ & $59,5 \mathrm{a}$ & $45,0 \mathrm{~d}$ & $78,5 \mathrm{~b}$ & $80,2 \mathrm{~b}$ & $42,7 \mathrm{a}$ \\
\hline Isoxaflutole & 75 g i.a. ha ${ }^{-1}$ & $29,0 \mathrm{~d}$ & $34,2 \mathrm{~d}$ & $10,0 \mathrm{~g}$ & $102,5 \mathrm{~b}$ & $115,5 \mathrm{a}$ & $40,2 \mathrm{a}$ \\
\hline Isoxaflutole & 112,5 g i.a. ha ${ }^{-1}$ & $35,0 \mathrm{c}$ & $37,2 \mathrm{c}$ & $15,0 \mathrm{f}$ & $141,0 \mathrm{a}$ & $143,7 \mathrm{a}$ & $32,5 \mathrm{~b}$ \\
\hline Chlorimuron & 7,5 g i.a. ha ${ }^{-1}$ & $5,0 \mathrm{e}$ & $10,5 \mathrm{f}$ & $35,0 \mathrm{e}$ & $140,5 \mathrm{a}$ & $135,5 \mathrm{a}$ & $31,5 \mathrm{~b}$ \\
\hline Chlorimuron & 12,5 g i.a. ha ${ }^{-1}$ & $5,0 \mathrm{e}$ & $15,0 \mathrm{e}$ & $80,0 \mathrm{a}$ & $68,7 \mathrm{~b}$ & $57,7 \mathrm{~b}$ & $46,3 \mathrm{a}$ \\
\hline Imazethapyr & 100 g i.a. ha ${ }^{-1}$ & $0,0 \mathrm{f}$ & $11,0 \mathrm{f}$ & $70,0 \mathrm{~b}$ & $62,2 \mathrm{~b}$ & $45,0 \mathrm{~b}$ & $42,8 \mathrm{a}$ \\
\hline Nicosulfuron & $40 \mathrm{~g}^{\text {i.a. }} \mathrm{ha}^{-1}$ & $0,0 \mathrm{f}$ & $10,0 \mathrm{f}$ & $50,0 \mathrm{c}$ & $75,5 \mathrm{~b}$ & $58,7 \mathrm{~b}$ & $39,8 \mathrm{a}$ \\
\hline $\mathrm{CV}(\%)$ & & 8,9 & 5,2 & 0,5 & 19,6 & 26,0 & 11,2 \\
\hline
\end{tabular}

Médias seguidas da mesma letra em cada coluna não diferem pelo teste Scott-Knott, $p \leq 0,05$.

O herbicida imazapyr (25 g i.a. ha $\left.{ }^{-1}\right)$ resultou em sintomas de injúria pouco pronunciados nas plantas de cedro-australiano, observados por leve amarelecimento de folhas. Na segunda avaliação de percentual de fitotoxicidade, as plantas não mais apresentavam sintomas visuais de injúria. Esse mesmo tratamento não resultou em perda de folhas, nem mesmo redução no teor de clorofila. $\mathrm{O}$ oxyfluorfen nas duas doses aplicadas proporcionou valores altos de percentual de fitotoxicidade aos 14 DAA (52 e 59\%). Embora tendo ocorrido recuperação das plantas aos 21 DAA (35 e 45\%), esses valores ainda são considerados altos. Além disso, ocorreu queda considerável de folhas, em ambas as avaliações. $\mathrm{O}$ isoxaflutole nas duas doses aplicadas resultou em valores intermediários de percentagem de fitotoxicidade nas duas primeiras avaliações, caracterizados por encarquilhamento de folhas. Aos 21 DAA, foi 
observada redução significativa desses valores, com recuperação das plantas. Esse fato se confirma ainda pelos valores de números de folhas obtidos na segunda avaliação aos 28 DAA, que não diferiram da testemunha sem aplicação. Os teores de clorofila também não diferiram da testemunha sem aplicação.

As duas doses de chlorimuron-ethyl causaram clorose acentuada das folhas de cedro. Houve progressão dos sintomas e, na avaliação aos 21 DAA, os valores obtidos foram 35 e $80 \%$, para a menor e a maior dose, respectivamente. A dose de $12,5 \mathrm{~g}$ i.a. ha ${ }^{-1}$ de chlorimuron causou redução considerável no número de folhas, em ambas as avaliações.

Da mesma forma, para os herbicidas imazethapyr e nicosulfuron, foi observada uma progressão dos sintomas de fitotoxicidade, que se fizeram notar com maior intensidade aos 21 DAA, havendo queda de folhas, em ambas as avaliações. Tiburcio et al. (2012) simularam a deriva de diferentes herbicidas em mudas de clones de eucalipto, verificando que é grande o risco de perda de produtividade caso haja intoxicação por deriva de nicosulfuron.

Os dados do experimento 2 relativos à matéria seca total, à matéria seca de raízes e caules e à área foliar do cedro-australiano, em função da aplicação das doses dos herbicidas, encontram-se na tabela 3.

Tabela 3. Matéria seca total (mst) (g.planta $\left.{ }^{-1}\right)$, matéria seca de raízes (msr) (g.planta $\left.{ }^{-1}\right)$, matéria seca de caules (msc) $\left(\right.$ g.planta $\left.{ }^{-1}\right)$ e área foliar (af) $\left(\mathrm{cm}^{2} \cdot\right.$ planta $\left.^{-1}\right)$, em função da aplicação das doses dos herbicidas sobre cedro-australiano.

Table 3. Total dry matter (g.plant $\left.{ }^{-1}\right)$, root dry matter $\left(\right.$ g.plant $\left.{ }^{-1}\right)$, stem dry matter (g.plant $\left.{ }^{-1}\right)$ and leaf area $\left(\mathrm{cm}^{2}\right.$.plant $\left.{ }^{-1}\right)$, in function of the application of herbicides on Australian cedar.

\begin{tabular}{|c|c|c|c|c|c|}
\hline & mst & msr & msc & af \\
\hline & $66,7 \mathrm{a}$ & $34,0 \mathrm{a}$ & $44,9 \mathrm{a}$ & $2905,9 \mathrm{a}$ \\
\hline $\begin{array}{l}\text { Testemunha } \\
\text { Imazapyr } \quad 25 \text { g i.a. ha }{ }^{-1}\end{array}$ & $\begin{array}{l}\text { Tratamentos } \\
\text { Testemunha } \\
\text { mazapvr } 25 \mathrm{~g} \text { i.a. ha }{ }^{-1}\end{array}$ & $56,5 \mathrm{a}$ & $32,7 \mathrm{a}$ & $38,0 \mathrm{a}$ & $2647,1 \mathrm{a}$ \\
\hline \multicolumn{2}{|c|}{ Oxyfluorfen $360 \mathrm{~g}$ i.a.ha ${ }^{-1}$} & $23,7 \mathrm{~b}$ & $10,4 \mathrm{~b}$ & $27,3 \mathrm{~b}$ & $1002,7 \mathrm{c}$ \\
\hline \multicolumn{2}{|c|}{ Oxyfluorfen 720 gi.a. ha ${ }^{-1}$} & $25,6 \mathrm{~b}$ & $10,2 \mathrm{~b}$ & $29,5 \mathrm{~b}$ & $1420,7 \mathrm{c}$ \\
\hline \multicolumn{2}{|c|}{ Isoxaflutole $\quad 75 \mathrm{~g}_{\text {i.a. }} \mathrm{ha}^{-1}$} & $33,0 \mathrm{~b}$ & $11,0 \mathrm{~b}$ & $43,0 \mathrm{a}$ & $2009,0 \mathrm{~b}$ \\
\hline Isoxaflutole & 112,5 g i.a. ha ${ }^{-1}$ & $25,2 \mathrm{~b}$ & $8,9 \mathrm{~b}$ & $34,1 \mathrm{a}$ & $2345,0 \mathrm{~b}$ \\
\hline \multicolumn{2}{|c|}{ Chlorimuron $7,5 \mathrm{~g}$ i.a. $\mathrm{ha}^{-1}$} & $19,2 \mathrm{~b}$ & $5,7 \mathrm{~b}$ & $27,6 \mathrm{~b}$ & 1944,7 b \\
\hline Chlorimuron & $12,5 \mathrm{~g}$ i.a. $\mathrm{ha}^{-1}$ & $13,0 \mathrm{~b}$ & $4,9 \mathrm{~b}$ & $15,4 \mathrm{~b}$ & $558,8 \mathrm{~d}$ \\
\hline \multirow{2}{*}{$\begin{array}{l}\text { Imazethapy } \\
\text { Nicosulfuron }\end{array}$} & 100 g i.a. ha $^{-1}$ & $14,1 \mathrm{~b}$ & $7,2 \mathrm{~b}$ & $16,0 \mathrm{~b}$ & $314,8 \mathrm{~d}$ \\
\hline & 40 g i.a. ha ${ }^{-1}$ & $20,5 \mathrm{~b}$ & $7,6 \mathrm{~b}$ & $22,0 \mathrm{~b}$ & $839,7 \mathrm{c}$ \\
\hline \multicolumn{2}{|l|}{$\mathrm{CV}(\%)$} & 19,7 & 24,7 & 28,1 & 28,9 \\
\hline
\end{tabular}

Médias seguidas da mesma letra em cada coluna não diferem pelo teste Scott-Knott, $\mathrm{p} \leq 0,05$.

Os resultados da tabela 3 confirmam aqueles avaliados anteriormente para o cedro. $\mathrm{O}$ tratamento menos fitotóxico foi o imazapyr. Os pesos da matéria seca total, da matéria seca de raízes e de caules e da área foliar não diferiram estatisticamente em relação à testemunha. Seguindo a mesma tendência resultante das avaliações de fitotoxicidade, os tratamentos com oxyfluorfen, isoxaflutole, chlorimuron, imazethapyr e nicosulfuron foram aqueles que causaram maior prejuízo para o crescimento e o desenvolvimento das plantas de cedro.

\section{CONCLUSÕES}

- Para o mogno-africano, os herbicidas imazapyr $\left(25 \mathrm{~g}\right.$ i.a. ha $\left.{ }^{-1}\right)$, oxyfluorfen $\left(360 \mathrm{~g}^{\text {i.a. }} \mathrm{ha}^{-1}\right)$ e chlorimuron-ethyl $\left(7,5 \mathrm{e} 12,5 \mathrm{~g}\right.$ i.a. $\left.\mathrm{ha}^{-1}\right)$ proporcionaram sintomas leves de fitotoxicidade. $\mathrm{O}$ oxyfluorfen $\left(720 \mathrm{~g}\right.$ i.a. ha $\left.{ }^{-1}\right)$, o imazethapyr $\left(100 \mathrm{~g}\right.$ i.a. ha $\left.{ }^{-1}\right)$ e o nicosulfuron $\left(40 \mathrm{~g}\right.$ i.a. ha $\left.{ }^{-1}\right)$ foram moderadamente fitotóxicos. O isoxaflutole $\left(75 \mathrm{e} 112,5 \mathrm{~g}\right.$ i.a. ha $\left.^{-1}\right)$ foi o herbicida mais fitotóxico. Para o cedro-australiano, o herbicida imazapyr $\left(25 \mathrm{~g}_{\text {i.a. }} \mathrm{ha}^{-1}\right)$ proporcionou os menores níveis de injúria. O oxyfluorfen, o isoxaflutole, o chlorimuron-ethyl, o imazethapyr e o nicosulfuron foram os mais fitotóxicos.

\section{AGRADECIMENTOS}

À Fundação de Amparo à Pesquisa do Estado de Minas Gerais e ao Conselho Nacional de Desenvolvimento Científico e Tecnológico, pelo apoio financeiro. 


\section{REFERÊNCIAS}

AGOSTINETTO, D.; TAROUCO, C. P.; MARKUS, C.; OLIVEIRA, E.; VAZ da SILVA, J. M. B.; TIRONI, S. P. Seletividade de genótipos de eucalipto a doses de herbicidas. Semina: Ciências Agrárias, Londrina, v. 31, n. 3, p. 585 - 598, 2010.

ALBINO, V. C. S; SÁ, V. A.; BUFALINO, L.; MENDES, L. M.; ALMEIDA, N. A. Avaliação de propriedades físico-mecânicas de painéis compensados de Toona ciliata. M. Roem. var. australis. Cerne, Lavras, v. 17, p. 103 - 108, 2010.

BALBINO, L. C.; BARCELOS, A. O.; STONE, L. F. Marco referencial em integração lavourapecuária-floresta. 1. ed. Brasília: Embrapa, 2011. v. 1. 132 p.

BRIGHENTI, A. M.; SOUZA SOBRINHO, F.; MARTINS, C. E.; ROCHA, W. S. D. Integração lavoura/pecuária/floresta. In: YAMAGUCHI, L. C. T.; MENDES, L. C. R.; LIMA, I. B.; ROGRIGUES, C. C.; RESENDE, M. L. Aspectos socioeconômicos e ambientais da produção de leite. Juiz de Fora, 2007. p. 107 - 124.

CARVALHO, A. M.; SILVA, B. T. B.; LATORRACA, J. V. Avaliação da usinagem e caracterização das propriedades físicas da madeira de mogno-africano (Khaya ivorensis A. Chev.). Cerne, Lavras, v.16, p. $106-114,2010$.

FERREIRA, L. R.; OLIVEIRA NETO, S. N.; FREITAS, F. C. L.; TIBÚRCIO, R. A. S.; VIANA, R. G.; MACHADO, M. S. Manejo de plantas daninhas na Integração Lavoura-Pecuária-Floresta. Informe Agropecuário, v. 31, p. 37 - 43, 2010.

FERREIRA, L. R.; MACHADO, M. S.; OLIVEIRA NETO, S. N.; FREITAS, F. C. L.; FERREIRA, G. L.; TUFFI SANTOS, L. D. Manejo de plantas daninhas em sistemas de ILPF. In: TUFFI SANTOS, L. D. Integração Lavoura-Pecuária-Floresta: potencialidades e técnicas de produção. 2012, p. 131 - 143.

MULLER, J. S. Sistemas agroflorestais com café (Coffea arabica L.) e cedro-australiano (Toona ciliata M. australis Bahadur) na Zona da Mata de Minas Gerais: estudo de caso. 50 f. Dissertação (Mestrado em Ciência Florestal) - Universidade Federal de Viçosa, Viçosa, 2004.

OLIVEIRA JR., R. S.; INOUE, M. H. Seletividade de herbicidas para culturas e plantas daninhas. In: OLIVEIRA JR., R. S.; CONSTANTIN, J.; INOUE, M. H. Biologia e manejo de plantas daninhas. Curitiba, 2011. p. 243 - 262.

PINHEIRO, A. L.; LANI, J. L.; COUTO, L. Cedro australiano: cultivo e utilização. 1 ed. Viçosa: NEPUT - UFV, 2003. v. 1.48 p.

PINHEIRO, A. L.; COUTO, L.; PINHEIRO, D. T.; BRUNETTA, J. M. F. C. Ecologia, silvicultura e tecnologia de utilização dos mognos-africanos (Khaya spp.). Viçosa: Sociedade Brasileira de Agrossilvicultura, 2011. 102 p.

RODRIGUES, B. N.; ALMEIDA, F. S. Guia de Herbicidas. 4. ed. Londrina: Edição dos autores, 1998. $648 \mathrm{p}$.

Guia de Herbicidas. 5. ed. Londrina: Edição dos autores, 2005. 592 p.

SÁ, V. A.; MENDES, L. M.; ALLAN LIMA, N. M. Manufatura de painéis cimento-madeira de cedroaustraliano (Toona ciliata. M. Roem. var. australis) de diferentes procedências e idades. Scientia Forestalis, Piracicaba, v. 38, p. 558 - 565, 2010.

SILVA, W.; SILVA, J. F.; CARDOSO, A. A.; BARROS, N. F. Tolerância de Eucalyptus spp. a diferentes herbicidas. Revista Árvore, Viçosa, v. 18, n. 3, p. 287 - 300, 1994.

Utilização de trifluralin 600 e do oxyfluorfen na cultura do Eucalyptus grandis Hill ex Maiden.

Revista Árvore, Viçosa, v. 19, n. 1, p. 1 - 17, 1995. 
SOCIEDADE BRASILEIRA DA CIÊNCIA DAS PLANTAS DANINHAS. Procedimentos para instalação e análise de experimentos com herbicidas. Londrina: SBCPD, 1995. 42 p.

TIBURCIO, R. A. S. Seletividade de herbicidas para eucalipto visando extensão de uso para sistemas agrossilviculturais. 55 f. Dissertação (Mestrado em Ciência Florestal) - Universidade Federal de Viçosa, Viçosa, 2010.

TIBURCIO, R. A. S.; FERREIRA, F. A.; MELO, C. A. D.; MEDEIROS, W. N.; PAES, F. A. S. V. Crescimento de mudas de clones de eucalipto submetidos à deriva simulada de diferentes herbicidas. Revista Árvore, Viçosa, v. 36, n. 1, p. 65 - 73, 2012.

TOLEDO, R. E. B.; ALVES, P. L. C. A., VALLE, C. F.; ALVARENGA, S. F. Comparação de custos de quatro métodos de manejo de Brachiaria decumbens Stapf em área de implantação de Eucaliptus grandis W. Hill ex Maiden. Revista Árvore, Viçosa, v. 20, n. 3, p. 319 - 330, 1996.

TOLEDO, R. E. B.; VICTORIA FILHO, R.; ALVES, P. L. C. A.; PITELLI, R. A.; CADINI, M. T. D. Efeitos da faixa de controle do capim-braquiária (Brachiaria decumbens) no desenvolvimento inicial de plantas de eucalipto. Planta Daninha, Viçosa, v. 18, n. 3, p. 383 - 393, 2000a.

TOLEDO, R. E. B.; VICTORIA FILHO, R.; PITELLI, R. A.; ALVES, P. L. C. A.; LOPES, M. A. F. Efeito de períodos de controle de plantas daninhas sobre o desenvolvimento inicial de plantas de eucalipto. Planta Daninha, Viçosa, v. 18, n. 3, p. 395 - 404, 2000b. 
FLORESTA, Curitiba, PR, v. 44, n. 4, p. 747 - 754, out. / dez. 2014. Brighenti, A. M.; Muller, M. D. 